\title{
MÖSSBAUER STUDY OF FERRIMAGNETIC ORDERING IN NICKEL FERRITE AND CHROMIUM-SUBSTITUTED NICKEL FERRITE
}

\author{
J. Chappert* and R. B. Frankel \\ National Magnet Laboratory, $\uparrow$ Massachusetts Institute of Technology, Cambridge, Massachusetts
}

(Received 24 July 1967)

\begin{abstract}
Mössbauer-effect measurements in external magnetic fields show that the magnetic structure of ferrimagnetic $\mathrm{NiFe}_{2} \mathrm{O}_{4}$ is the collinear Neel type. $\mathrm{NiFe}_{0.3} \mathrm{Cr}_{1.7} \mathrm{O}_{4}$ is shown to have a triangular structure with $\theta_{A}=20 \pm 10^{\circ}$ and $\theta_{B}=50 \pm 4^{\circ}$.
\end{abstract}

In a recent Letter, ${ }^{1}$ Kedem and Rothem have presented Mössbauer data which they propose is evidence for a Yafet-Kittel triangular spin arrangement ${ }^{2}$ in the ferrimagnetic spinel $\mathrm{Ni} \mathrm{Fe}_{2} \mathrm{O}_{4}$. Their conclusions are in sharp disagreement with the results of susceptibility measurements by Jacobs, ${ }^{3}$ who proposed a Neel collinear mod$\mathrm{el}^{4}$ for this material. We report here Mössbauer measurements in external magnetic fields which provide conclusive evidence for the Néel model and thus support the conclusions drawn by Jacobs. In addition, we show that the chromium substituted ferrite $\mathrm{NiFe}_{0.3} \mathrm{Cr}_{1.7} \mathrm{O}_{4}$ is consistent with a Yafet-Kittel model.

Many of the magnetic properties of the ferrimagnetic spinel compounds $\mathrm{M}^{2+} \mathrm{N}_{2}{ }^{3+} \mathrm{O}_{4}$ are well understood on the basis of the Néel collinear model. However, for spinels with large amounts of chromium, the spontaneous magnetization is lower than the expected from this model and is usually interpreted in terms of the Yafet-Kittel triangular arrangement in which each tetrahedral $A$ and octahedral $B$ sublattice is divided into two sub-sublattices; the resultant moments of the two triangular sublattices are antiparallel. Experimental evidence for the Yafet-Kittel model has been established by high-field susceptibility measurements ${ }^{3,5}$ and neutron-diffraction experiments. ${ }^{6,7}$ Previous $\mathrm{nmr}^{8}$ and Mössbauer ${ }^{9,10}$ studies of $\mathrm{NiFe}_{2} \mathrm{O}_{4}$ indicate two different hyperfine fields, corresponding to the iron ions on the $A$ sites and the $B$ sites. However, Kedem and Rothem ${ }^{1}$ have concluded, mainly from the width of the Mössbauer lines, that there are four hyperfine fields and that this observation constituted experimental evidence for the Yafet-Kittel model.

Our samples were made by firing mixed oxides including $\mathrm{Fe}_{2} \mathrm{O}_{3}$ enriched in $\mathrm{Fe}^{57}$ in a platinum crucible at $1200^{\circ} \mathrm{C}$ in air for ten hours; the resulting product was then ground to a pow- 
der, heated in a nitrogen atmosphere at $1200^{\circ} \mathrm{C}$ for 12 hours, and then allowed to cool slowly. $\mathrm{X}$-ray diffraction analysis confirmed the spinel structure and the absence of other phases.

The Mössbauer experiments were performed using a conventional constant acceleration electromechanical drive system together with a multichannel analyzer for collecting and storing the data. The magnetic field was produced by a $\mathrm{Nb}_{3} \mathrm{Sn}$ superconducting solenoid operating in the persistent mode up to $75 \mathrm{kOe}$.

Most of the experiments were carried out with the magnetic field applied along the $\gamma$ ray propagation direction. If the moments are collinear with the field, the polarization conditions require the disappearance of the $\Delta m=0$ lines in the hyperfine pattern. Figure 1 (a) shows the results for $\mathrm{Ni} \mathrm{Fe}_{2} \mathrm{O}_{4}$ at $4.2^{\circ} \mathrm{K}$. The zero field spectrum indicates two hyperfine field $\mathrm{s}^{1,9,10}$; the widths of the lines are greater than those obtained with an $\alpha-\mathrm{Fe}_{2} \mathrm{O}_{3}$ absorber, but this is expected in a powder

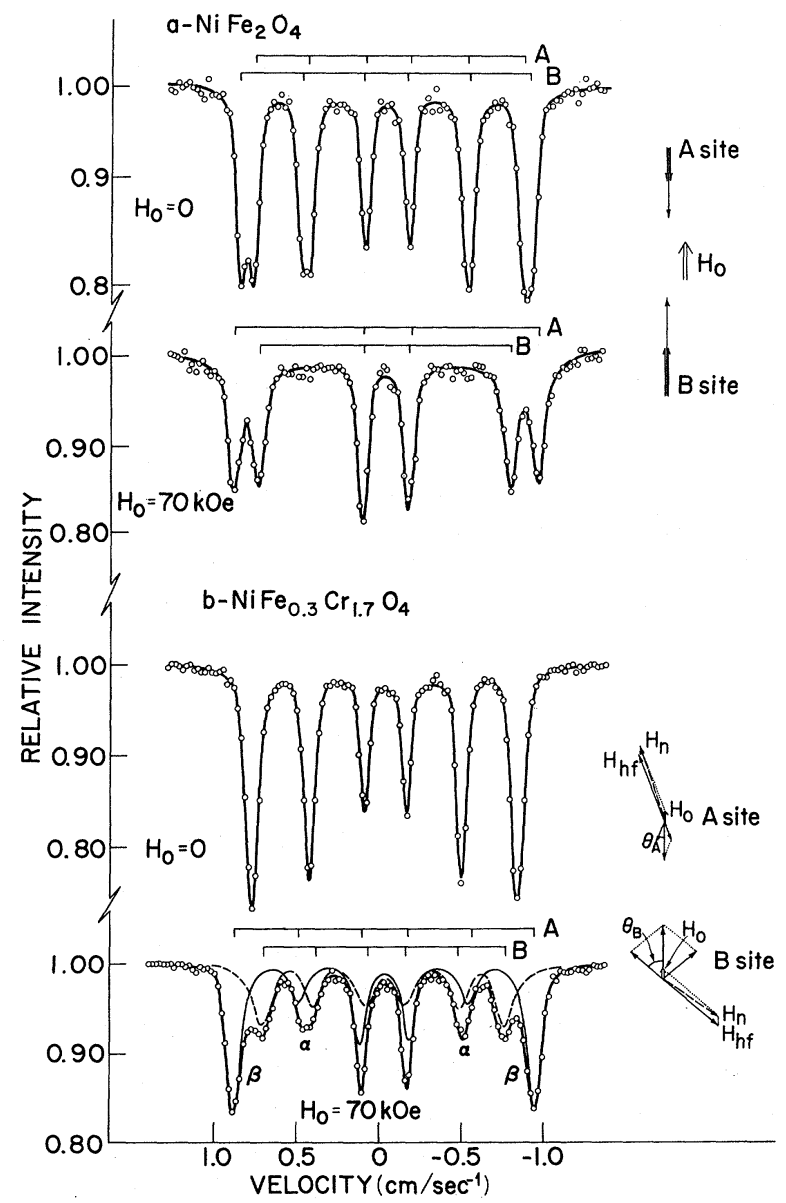

FIG. 1. Mössbauer spectra at $T=4.2^{\circ} \mathrm{K}$. (a) $\mathrm{NiFe}_{2} \mathrm{O}_{4}$. (b) $\mathrm{NiFe}_{0.3} \mathrm{Dr}_{1,7} \mathrm{O}_{4}$. sample. The effect of applying a longitudinal field to the $\mathrm{NiFe}_{2} \mathrm{O}_{4}$ sample is also shown in Fig. 1(a); one observes the disappearance of the $\Delta m=0$ lines at about $H_{0}=12 \mathrm{kOe}$, and a further increase of the external field splits the outer $\Delta m= \pm 1$ lines into doublets of equal intensity, corresponding to the spin-up and spin-down sublattices. The measured fields at the nuclei $H_{n}$ for the sublattices $T=4.2^{\circ} \mathrm{K}$ and $H_{0}=70 \mathrm{kOe}$ are $-574 \pm 5 \mathrm{kOe}(A$ site $)$ and $-477 \pm 5 \mathrm{kOe}(B$ site), and using the relation

$$
H_{n}(Z)=H_{\mathrm{hf}}(Z) \pm H_{0}, \quad Z=(A, B),
$$

where $H_{\mathrm{hf}}(Z)$ are the hyperfine fields, we find $H_{\mathrm{hf}}(A)=-504 \pm 5 \mathrm{kOe}$ and $H_{\mathrm{hf}}(B)=-547 \pm 5 \mathrm{kOe}$ in agreement with the hyperfine fields measured at $H_{0}=0:-506 \pm 5 \mathrm{kOe}(A)$ and $-548 \pm 5 \mathrm{kOe}$ $(B)$. These observations constitute definitive evidence for the Néel model in $\mathrm{NiFe}_{2} \mathrm{O}_{4}$. A 0.005in. -thick $\mathrm{NiFe}_{2} \mathrm{O}_{4}$ single crystal, the plane of which is perpendicular to the [100], was also studied. Application of a small transverse field $(1.25 \mathrm{kOe})$ in the plane of the absorber fully aligned the moments as evidenced by the relative intensities of the $\Delta m=0$ and $\Delta m= \pm 1$ hyperfine lines, indicating very low anisotropy. A portion of the crystal was crushed to make a polycrystalline absorber and was studied in high external magnetic fields; the results were identical with the powder spectra.

The hyperfine spectra of $\mathrm{NiFe}_{0.3} \mathrm{Cr}_{1.7} \mathrm{O}_{4}$ are shown in Fig. 1(b). It has been generally supposed ${ }^{11,12}$ that $\mathrm{Fe}^{3+}$ ions are situated on the $A$ sites. However, the 70-kOe spectrum [ Fig. $1(\mathrm{~b})]$ indicates that approximately $\frac{1}{3}$ of the $\mathrm{Fe}^{3+}$ ions are on the $B$ sites. The hyperfine fields for the two sites overlap at $4.2^{\circ} \mathrm{K}$ in zero external field and have the value $-498 \pm 5 \mathrm{kOe}^{13}$ Unlike the $\mathrm{Ni} \mathrm{Fe}_{2} \mathrm{O}_{4}$, at $H_{0}=70 \mathrm{kOe}$ the $\Delta m=0$ lines [lines $\alpha$ in Fig. 1(b)] are still relatively intense and the $\Delta m= \pm 1$ lines $(\beta)$ do not correspond to Eq. (1). Assuming no large magnetocrystalline anisotropy, ${ }^{3}$ this may be explained by a Yafet-Kittel arrangement in which the spins on each sublattice $Z(Z=A, B)$ make an angle $\theta_{Z}$ with the external field direction. For this model, the field at the nucleus $H_{n}(Z)$ in an external field $H_{0}$ is given by

$$
H_{n}(Z)=\left(H_{0}{ }^{2}+H_{\mathrm{hf}}{ }^{2}-2 H_{0}{ }_{\mathrm{hf}} \cos \theta{ }^{1 / 2} .\right.
$$

From the observed spectrum we find $\theta_{A}=20$ $\pm 10^{\circ}$ and $\theta_{B}=50 \pm 4^{\circ} .{ }^{14}$ Using these values of 
$\theta_{A}$ and $\theta_{B}$ we are able to calculate the expected values of the relative intensities of the $\Delta m$ $=0$ lines. These values ( $x=0.3$ for the $A$ site, 1.6 for the $B$ site) are in good agreement with the fit indicated for the $70-\mathrm{kOe}$ spectrum of Fig. 1(b). Taking into account the presence of iron in the $B$ site, one finds that the spontaneous moment calculated for such a model is equal to $1.3 \pm 0.5 \mu_{\mathrm{B}}$, which is significantly lower than the moment expected from a Néel model $\left(3.2 \mu_{\mathrm{B}}\right){ }^{15}$ Furthermore, the $B$ site moment is dominant in agreement with susceptibility data. ${ }^{3}$ We note that the observation of only two hyperfine fields is consistent with both the Yafet-Kittel as well as the Neel model and is therefore not sufficient to distinguish between these models.

We conclude that the magnetic structure of ferrimagnetic $\mathrm{NiFe}_{2} \mathrm{O}_{4}$ is of the collinear Néel type. An example of a Yafet-Kittel structure is shown for the chromium substituted $\mathrm{NiFe}_{0.3} \mathrm{Cr}_{1.7} \mathrm{O}_{4}$.

We thank Professor A. J. Freeman for his interest and stimulation and Professor E. F. Bertaut, Dr. N. A. Blum, Dr. S. Foner, and Dr. I. S. Jacobs for useful discussions. We gratefully acknowledge Mr. J. E. C. Williams of the National Magnet Laboratory for design and construction of the superconducting magnet.

* On leave from the Centre d'Etudes Nucléaires, Grenoble, France. Supported by Commissariat à 1'Energie Atomique, France.

†Supported by the U. S. Air Force Office of Scientific Research.

${ }^{1}$ D. Kedem and T. Rothem, Phys. Rev. Letters 18, 165 (1967).
${ }^{2}$ Y. Yafet and C. Kittel, Phys. Rev. 87, 290 (1952).

${ }^{3}$ I. S. Jacobs, J. Phys. Chem. Solids 15, 54 (1959).

${ }^{4}$ L. Néel, Ann. Phys. (Paris) 3 , 137 (1948).

${ }^{5}$ P. L. Edwards, Phys. Rev. 116, 294 (1959).

${ }^{6}$ E. Prince, Acta Cryst. 10, 554 (1957).

${ }^{7}$ R. Nathans, S. J. Pickart, and A. Miller, Bull. Am. Phys. Soc. $\underline{6}, 54$ (1961).

${ }^{8} \mathrm{H}$. Abe, M. Matsuura, H. Yasuoka, A. Harai, T. Hashi, T. Fukuyama, J. Phys. Soc. Japan 18, 1400 (1963).

${ }^{9}$ V. I. Gol'danskii, V. F. Belov, M. N. Devisheva, and V. A. Trukhtanov, Zh. Eksperim. i Teor. Fiz. $\underline{49}$, 1681 (1965) [translation: Soviet Phys.-JETP $\underline{22}, 1149$ (1966)].

${ }^{10}$ J. P. Morel, J. Phys. Chem. Solids 28, 629 (1967).

${ }^{11}$ P. K. Baltzer and P. J. Wojtowicz, J. Appl. Phys. Suppl. 30, 27S (1959).

${ }^{12} \mathrm{~J}$. B. Goodenough, Magnetism and the Chemical Bond (John Wiley \& Sons, Inc., New York, 1963).

${ }^{13}$ The temperature dependence of the Mössbauer spectrum of $\mathrm{NiFe}_{0.3} \mathrm{Cr}_{1.7} \mathrm{O}_{4}$ shows evidence of relaxation effects in the paramagnetic and in the ferrimagnetic state [I. Nowik and H. H. Wickman, Phys. Rev. Letters 17, $949(1966)]$ from $120^{\circ} \mathrm{K}$ to $\simeq 500^{\circ} \mathrm{K}\left(T_{N} \simeq 280^{\circ} \mathrm{K}\right)$. Above $120^{\circ} \mathrm{K}$, the hyperfine lines broaden and we observe the appearance of a quadrupole doublet which increases in intensity as $T$ increases. The presence of a quadrupole splitting $(0.40 \pm 0.05 \mathrm{~mm} / \mathrm{sec})$ can be attributed to some local distortion of the crystallographic structure mainly for the $A$ sites [T. R. McGuire and S. W. Greenwald, Bull. Am. Phys. Soc. $\underline{3}$, 43 (1958)].

${ }^{14}$ Susceptibility measurements (Ref. 3 ) require an angle on the $B$ site.

${ }^{15}$ The moment derived from magnetization curves is about $0.5 \mu_{\mathrm{B}}$ [Ref. 3 and T. R. McGuire and S. W. Greenwald, Bull. Am. Phys. Soc. $\underline{2}, 22$ (1957)]. However, it has recently been shown [F. Van der Woude, G. A. Sawatsky, and A. H. Morrish, Bull. Am. Phys. Soc. 12, 724 (1967)] that the distribution of the cations in the $A$ and $B$ sites is related to the method of preparation and heat treatment. For a sample with iron in tetrahedral sites only, but with the same $\theta_{A}$ and $\theta_{B}$ as for our sample, the theoretical moment would be $1.0 \pm 0.5 \mu_{\mathrm{B}}$. 